\title{
Discrimination of Interictal and Preictal States Using Wavelet Analysis on EEG Signals of Patients with Intractable Convulsions
}

\author{
Jade B. Kill* Patrick Marques Ciarelli** \\ Klaus F. Côco** Anselmo F. Neto**
*Programa de Pós-Graduação em Engenharia Elétrica, Universidade Federal do Espírito Santo, ES 29075-910 BR (Tel: +55 27 99753-9969; e-mail: jadebkill@ hotmail.com).
** Departamento de Engenharia Elétrica, Universidade Federal do Espirito Santo, ES 29075-910 BR (e-mail: \{patrick.ciarelli, klaus.coco,\}@ufes.br; anselmo@ele.ufes.br).

\begin{abstract}
The discrimination of the interictal and preictal states contributes to the construction of an efficient system of seizure prediction. Here, we performed the classification of the interictal and preictal states for EEG signals of the scalp. The energies of the levels obtained by the signal decomposition of the Wavelet Discrete Transform were used as features for classification. The kNN and SVM classifiers were used in the analysis of the individual EEG channels, which gave indications that the occipital lobe region channels are the most relevant to differentiate between the interictal and preictal states. Using these channels, the classification into two states achieved accuracy of $97.29 \%$, sensitivity of $96.25 \%$ and specificity of $98.33 \%$. In addition, the different frequency ranges obtained by Wavelet for the classification were analyzed, and it was observed that the range of $32 \mathrm{~Hz}$ to $128 \mathrm{~Hz}$ presented greater relevance in the task.
\end{abstract}

Keywords: Epilepsy; Electroencephalogram; Wavelet; Prediction; Preictal; Interictal.

\section{INTRODUCTION}

Epilepsy is a chronic brain disorder characterized by repeated seizures. There are more than 50 million people worldwide who are diagnosed with epilepsy and this neurological disease is considered one of the most common in the world. However, approximately $80 \%$ of this population, who live in low and middle-income countries, do not receive adequate treatment (Who, 2019).

People with intractable epilepsy often lack the knowledge of when a seizure will occur. This uncertainty can lead to difficulties in carrying out daily activities such as driving, working, or even socializing, and puts patients at higher risk for injuries. By presenting information about when a seizure is likely to occur, the patient will have some control over the seizures and prevent possible accidents, as well as being very useful in preventing seizures that can be avoided by medication (Williamson et al., 2012; Usman, Usman and Fong, 2017).

Studies on changes in brain dynamics monitored by electroencephalography (EEG) have been promising for predicting seizures (Freestone, Karoly and Cook, 2017). Researches have demonstrated that EEG signals can be used for procedures of detection and prediction of seizures (Sayeid et al., 2016).

The EEG records can be intracranial or scalp. Although intracranial recordings are less susceptible to noise and artifacts (e.g. body motion and electrodes movements), there is a preference for the EEG scalp because it is a non-invasive technique (Tsiouris et al., 2018).

Several seizure prediction methods have been applied in the last years, but many of them still have low sensitivity and high false prediction rate (above $0.15 / \mathrm{h}$ ). Moreover, the ideal range of true and false predictions is still not very well defined, as it is related to the amount of convulsions of each patient (Sharif and Jafari, 2017).

Research on prediction of seizures usually study the differences between interictal states (period between seizures) and preictais (period immediately preceding a seizure). Other approaches have sought the best way to combine characteristics to generate accurate and reliable information about the prediction (Williamson et al., 2012).

The Wavelet Transform (WT) has been used to extract features on the dynamic structure of EEG signals, from the decomposition of EEG signals into levels (Kocadagli and Langari, 2017). Many researchers suggest the detection of epilepsy, or epileptic seizures, using WT of EEG signals. Studies with the use of this method in cases of prediction of epileptic seizures and of discrimination between interictal and preictal states have been very promising in recent years (Gadhoumi, Lina and Gotman, 2012; Sayeid et al., 2016).

Gadhoumi, Lina and Gotman (2012) proposed a method based on the analysis of high frequency activity of intracerebral activity. The researchers used wavelet energy and entropy to discriminate between the interictal and preictal states. However, for this method to detect the differences 
effectively, it is necessary that at least one channel is in the region is where the dynamics of the preictal and interictal state are different. In addition, the method does not have high performance for all six patients, with a chance of presenting poor results for other patients.

Song and Zhang (2016) assess whether sample entropy can be used to discriminate the interictal and preictal states of 6 intracranial EEG channels. For classification using the support vector machine (SVM) classifier, it obtained accuracy of approximately $84 \%$, indicating that the method can still be improved.

The mechanisms generating seizure may be specific to each patient diagnosed with epilepsy. Therefore, it is seldom possible to be sure if these algorithms will work for a given patient when applied prospectively (Freestone, Karoly and Cook 2017). It has still been challenging to correctly define the problems related to the prediction of seizure and this generates a lack of confidence in machine learning algorithms that have been applied in recent years. This confirms that there is an unmet need for a system that provides early warning of seizure, which can support new approaches to treatment and improve the patient's quality of life (Kiral-Kornek et al., 2018). More signal analysis are needed to achieve more effective results and methods applied to all patients.

In addition, previous studies indicate that seizure precursors appear only in certain distinct channels and may facilitate the use of specific devices and early identification of the seizure (Mormann et al., 2005; Chu et al., 2017). Chu et al. (2017) used two EEG databases, with a total of 16 patients. The researchers performed channel selection and gave more relevance to the channel that presented better performance. In the classification of the interictal and preictal states, they reached sensitivity of $86.67 \%$, but with few training seizures.

In this study we present an approach to classify the interictal and preictal states of patients with intractable epilepsy as an important step in predicting seizures. The CHB-MIT data set, collected from patients with intractable seizures, was used using scalp EEG. This data set has recently been studied for the prediction of epileptic seizures, but its use is still quite challenging due to the heterogeneity of patients and the low number of studies that used it to discriminate states.

To perform frequency-time analysis of the signal in each state, the Discrete Wavelet Transform (DWT) was used to extract the characteristics of the signals. In addition, a channel analysis was performed to evaluate the most relevant EEG channels that achieved the highest performance in the classification.

From the pre-selection of the channels it is possible to identify regions of the brain that best represents the differences of the cerebral dynamics between the preictal and interictal states. Identifying regions with more discriminating signals is an important step in developing better methods and devices that can be used to predict seizures, which are cheaper and less visually perceptible.
This work has the following structure. Section 2 describes the methods used in data pre-processing and model validation, the performance measures applied in the experiments and the description of the methodology to classify. In Section 3 we discuss the results obtained and important observations. Finally, we conclude in Section 4.

\section{METHODS}

\subsection{Discrete wavelet transform}

The DWT is used in signal preprocessing to represent frequency characteristics through its coefficients (Faust et al., 2015). This technique is sensitive to changes in EEG signals, which are difficult to detect by analyzing the signal only visually (Faust et al., 2015).

The DWT algorithm decomposes a given signal into approximation coefficients and details to obtain a first level of decomposition. The approximation coefficients at all levels are further decomposed into the next approach level and detail coefficients (Faust et al., 2015). The definition of the discrete transform of periodic signals is described in more detail by Gubner and Chang (1995).

The shape and frequency of the signal are related to the mother wavelet, which is usually an orthonormal basis function. The choice of the most similar mother wavelet with the analyzed signal is important to obtain a better decomposition. The Daubechies, db4, wavelet is most commonly used in DWT researches on EEG signals, since it has the highest classification accuracy (Khan, Gotman, 2003; Saab, Gotman, 2005; Faust et al., 2015; Li, Chen and Zhang, 2017).

In this paper, the DWT was used to decompose time windows of 10 seconds, using all 23 channels. The Daubechies db4 wavelet was the mother wavelet applied to decompose the signals into 5 levels.

\subsection{Energy}

For the Daubechies wavelet, which forms an orthogonal basis in the time-frequency plane, the sum of the squares of the coefficients of the wavelet series is the signal energy (Khan, Gotman, 2003). The energy for each sub-band obtained by the DWT is given as:

$e_{s b}=\sum_{i=1}^{n} C_{i}^{2} \frac{\Delta s}{n}$

where $n$ is the number of coefficients $C$ obtained by the DWT at the level $s b$, with sampling interval $\Delta s$.

\subsection{Classification Method}

The classification of EEG signals has been quite challenging in the field of machine learning and signal processing (Richhariya, Tanveer, 2018). 
This study consists of preprocessing the data using the DWT on EEG signals and classifying them using kNN (k-nearest neighbors) (Cover, Hart, 1967) and SVM (Burges, 1998). The optimum value of $\mathrm{k}$ obtained in the calibration of the classification algorithm $\mathrm{kNN}$ was equal to 3 and the Euclidean distance was used as distance metric. The values tested were only the odd ones, so as not to cause a tie in the classification. SVM is a powerful EEG signal classification technique capable of obtaining a globally optimal solution (Richhariya, Tanveer, 2018). For the development of the SVM classifier, we fit into a linear model and parameter $\mathrm{C}$ equals 4 .

The wavelet transform is one of the most widely used methods for extracting EEG data features (Richhariya, Tanveer, 2018). Non-overlapping sliding windows of 10 seconds were analyzed using the energy calculated of each level obtained from the DWT. In our approach we used db4 wavelet with 5 levels of decomposition. The features are used in the classifiers to identify differences between preictal and interictal states.

\subsection{K-fold Cross Validation}

To evaluate the generalization capacity of the classification model, the $\mathrm{k}$-fold cross-validation technique was used, with $k$ equal to 5 . The $\mathrm{k}$-fold cross-validation is normally performed to ensure the generalization of a classification model. In this method, the data set $\mathbf{X}$ is randomly divided into $k$ mutually exclusive, and approximately equal size, subsets (Kohavi, 1995).

For training, $k-1$ subsets are used, and the remainder is used as test set. Thus, performance metrics are calculated iteratively by $k$ times, where in each iteration a different subset is used as test set. After cross-validation, the mean of the results is calculated as final result (Kohavi, 1995).

\subsection{Performance Testing}

The classification performance is evaluated in the test data set in terms of accuracy (Acc), sensitivity (S) and specificity (Sp) which are defined in Equations (1), (2) and (3):

$$
\begin{aligned}
& A c c=\frac{T P+T N}{T P+F P+T N+F N} 100 \% \\
& S=\frac{T P}{T P+F N} 100 \% \\
& S p=\frac{T N}{F P+T N} 100 \%
\end{aligned}
$$

where TP (True Positive) are the samples correctly classified as positive, that is, they actually belong to the class that the classifier chose, FP (False Positive) and FN (False Negative) are the samples erroneously classified by the model and TN (True Negative) are the samples that do not belong to a certain class and are classified correctly.

\section{EXPERIMENTAL DESIGN AND RESULTS}

\subsection{EEG database}

The CHB-MIT data set (available online: https://www.physionet.org/pn6/chbmit/), which contains EEG signal records acquired from different regions of the scalp, was used in our experiments to discriminate between the interictal and preictal states. Fig. 1 shows segments of an EEG signal of the interictal and preictal state. This database was collected from 23 pediatric patients with intractable seizures at the Boston Children's Hospital. The times of onset of the seizures for the different registries were noted previously.
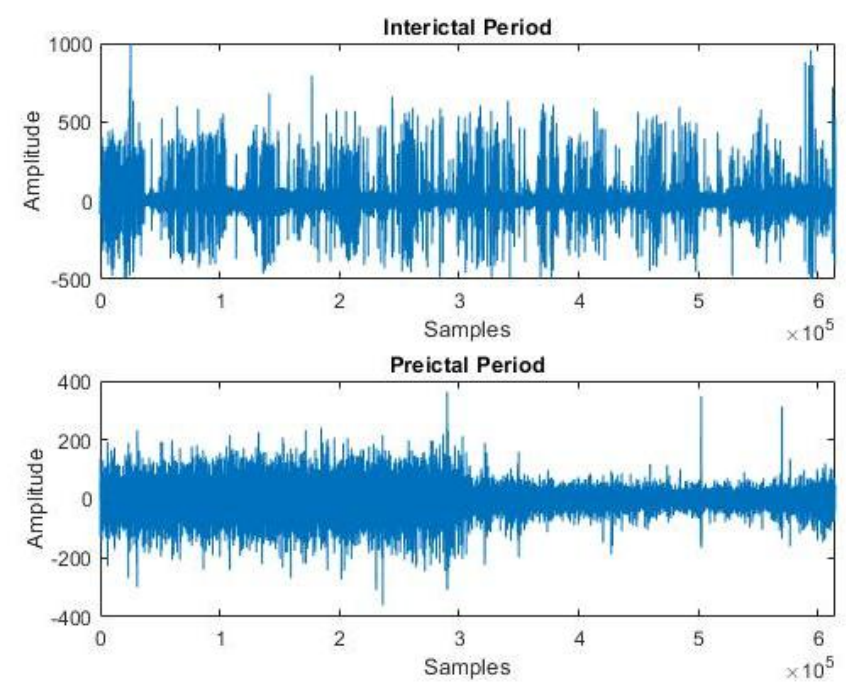

Figure 1 - Examples of periods referring to EEG signals of only 1 channel, lasting 40 minutes.

All signals were sampled at 256 samples per second. The signs studied present 23 EEG channels, with recordings of 1, 2 or 4 hours of digitized EEG signals. For these recordings, the International 10-20 system was used for positions and nomenclature of the EEG electrodes. Thus, channels 1 through 23 are respectively: $\mathrm{FP}_{1}-\mathrm{F}_{7}, \mathrm{~F}_{7}-\mathrm{T}_{7}, \mathrm{~T}_{7}-\mathrm{P}_{7}, \mathrm{P}_{7}-\mathrm{O}_{1}, \mathrm{FP}_{1^{-}}$ $\mathrm{F}_{3}, \mathrm{~F}_{3}-\mathrm{C}_{3}, \mathrm{C}_{3}-\mathrm{P}_{3}, \mathrm{P}_{3}-\mathrm{O}_{1}, \mathrm{FP}_{2}-\mathrm{F}_{4}, \mathrm{~F}_{4}-\mathrm{C}_{4}, \mathrm{C}_{4}-\mathrm{P}_{4}, \mathrm{P}_{4}-\mathrm{O}_{2}, \mathrm{FP}_{2}-\mathrm{F}_{8}$, $\mathrm{F}_{8}-\mathrm{T}_{8}, \mathrm{~T}_{8}-\mathrm{P}_{8}, \mathrm{P}_{8}-\mathrm{O}_{2}, \mathrm{Fz}-\mathrm{Cz}, \mathrm{Cz}-\mathrm{Pz}, \mathrm{P}_{7}-\mathrm{T}_{7}, \mathrm{~T}_{7}-\mathrm{FT}_{9}, \mathrm{FT}_{9}-\mathrm{FT}_{10}$, $\mathrm{FT}_{10}-\mathrm{T}_{8}$ e $\mathrm{T}_{8}-\mathrm{P}_{8}$.

To select the interictal states, 40-minute intervals were selected, approximately 4 hours before or after epileptic seizures (ictal state) (Truong et al., 2018). For the preictal, intervals of $40 \mathrm{~min}$, up to 1 second before the ictal, were selected.

The total recording time used in the analyzes was approximately 19.3 hours of interictal and 19.3 hours of preictal.

\subsection{Channel analysis}

The knowledge of the most relevant channels in the classification process gives us the best location on the scalp 
that discriminates between the interictal and preictal states. The decomposition of the EEG signals by DWT into 5 levels returned 6 frequency ranges, as can be seen in Table 1 .

At first, each channel was analyzed individually, in the task of performing the classification of the signal in interictal and preictal. The results of this analysis, in terms of the performance measures, are presented in Table 2, for the kNN and SVM classifiers.

Table 1. Frequency bands of EEG signal using five level decomposition

\begin{tabular}{|c|c|c|}
\hline Levels & Sub-bands & Frequency range $(\mathrm{Hz})$ \\
\hline 1 & D1 & $64-128$ \\
\hline 2 & D2 & $32-64$ \\
\hline 3 & D3 & $16-32$ \\
\hline 4 & D4 & $8-16$ \\
\hline 5 & D5 & $4-8$ \\
\hline 5 & A5 & $0-4$ \\
\hline
\end{tabular}

Table 2. Performance of $\mathrm{kNN}$ and SVM classifiers in relation to individually analyzed channels

\begin{tabular}{|c|c|c|c|c|c|c|}
\hline Channels & $\begin{array}{c}\text { Acc } \\
\text { kNN } \\
(\%)\end{array}$ & $\begin{array}{c}\text { Acc } \\
\text { SVM } \\
(\%)\end{array}$ & $\begin{array}{c}\text { SNN } \\
\text { (\%) }\end{array}$ & $\begin{array}{c}\text { SVM } \\
\text { SV })\end{array}$ & $\begin{array}{c}\text { Sp } \\
\text { kNN } \\
(\%)\end{array}$ & $\begin{array}{c}\text { Sp } \\
\text { SVM } \\
(\%)\end{array}$ \\
\hline 1 & 76.25 & 71.04 & 77.50 & 65.41 & 75.00 & 76.67 \\
\hline 2 & 69.79 & 62.71 & 80.00 & 50.42 & 59.58 & 75.00 \\
\hline 3 & 79.17 & 84.38 & 87.08 & 90.00 & 71.25 & 78.75 \\
\hline 4 & 90.42 & 90.83 & 90.00 & 88.33 & $\mathbf{9 0 . 8 3}$ & 93.33 \\
\hline 5 & 74.38 & 69.58 & 76.25 & 56.25 & 72.50 & 82.92 \\
\hline 6 & 77.29 & 73.33 & 78.33 & 69.17 & 76.25 & 77.50 \\
\hline 7 & 77.50 & 69.17 & 70.42 & 89.58 & 84.58 & 48.75 \\
\hline 8 & 79.17 & 75.00 & 77.92 & 92.50 & 80.42 & 57.50 \\
\hline 9 & 72.71 & 70.21 & 77.08 & 92.50 & 68.33 & 47.92 \\
\hline 10 & 80.00 & 80.42 & 83.33 & 90.00 & 76.67 & 70.83 \\
\hline 11 & 71.46 & 64.79 & 75.42 & 92.92 & 67.50 & 36.67 \\
\hline 12 & 88.12 & 90.42 & 87.08 & 86.25 & 89.17 & $\mathbf{9 4 . 5 8}$ \\
\hline 13 & 78.33 & 75.00 & 81.25 & 67.92 & 75.42 & 82.08 \\
\hline 14 & 71.25 & 61.25 & 79.58 & 58.75 & 62.92 & 63.75 \\
\hline 15 & 82.71 & 84.38 & 90.83 & 87.08 & 74.58 & 81.67 \\
\hline 16 & $\mathbf{9 2 . 9 2}$ & $\mathbf{9 3 . 7 5}$ & $\mathbf{9 5 . 4 2}$ & $\mathbf{9 4 . 5 8}$ & 90.42 & 92.92 \\
\hline 17 & 58.75 & 64.58 & 63.33 & 86.67 & 54.17 & 42.50 \\
\hline 18 & 73.75 & 69.37 & 75.83 & 93.33 & 71.67 & 45.42 \\
\hline 19 & 81.25 & 84.58 & 88.33 & 90.83 & 74.17 & 78.33 \\
\hline 20 & 72.92 & 71.04 & 85.00 & 82.08 & 60.83 & 60.00 \\
\hline 21 & 67.92 & 58.54 & 68.75 & 93.75 & 67.08 & 23.33 \\
\hline 22 & 67.92 & 71.25 & 75.42 & 77.08 & 60.42 & 65.42 \\
\hline 23 & 81.46 & 84.67 & 89.58 & 86.67 & 73.33 & 81.67 \\
\hline
\end{tabular}

When analyzing the obtained results, it was noticed that the most relevant channels were 4 (P7-O1), 12 (P4-O2) and 16
(P8-O2). By mapping their location on the scalp, was observed to be in the lobe occipital region (4 (P7-O1), 8 (P3$\mathrm{O} 1), 12(\mathrm{P} 4-\mathrm{O} 2)$ and $16(\mathrm{P} 8-\mathrm{O} 2))$.

Based on this observation, other experiments were done, this time comparing the use of all channels with those of the channels of the occipital lobe region $(4,8,12$ and 16). It was also verified the interference in the classification of the right and left sides. For the channels 12 and 16, which are located further on the right side, and for the channels 4 and 8 , which are more on the left side. These results are shown in Table 3.

As we can see in Table 3, the four selected channels achieved the best results, with accuracy of $97.29 \%$, sensitivity $96.25 \%$ and specificity with $98.33 \%$, when using the SVM classifier. The SVM classifier that used all channels also resulted in good performances, but for the purpose of creating a seizure alarm device, the use of 23 channels would make it difficult for the patient's daily activities. Besides this information, it was verified that the $\mathrm{kNN}$ classifier obtained its best accuracy and specificity when using the channels 4,12 and 16 . When analyzing the effect of the right and left sides in the classification, it was observed, for both classifiers and measures, right side was slightly better than left side.

The results achieved in Table 2 and 3 indicate the occipital lobe region has a great discriminative power to distinguish between the interictal and preictal states.

Table 3. Performance of the kNN and SVM classifiers in relation to the selected channel sets and all channels

\begin{tabular}{|c|c|c|c|c|c|c|}
\hline Channels & $\begin{array}{c}\text { Acc } \\
\text { kNN } \\
(\%)\end{array}$ & $\begin{array}{c}\text { Acc } \\
\text { SVM } \\
(\%)\end{array}$ & $\begin{array}{c}\text { SNN } \\
(\%)\end{array}$ & $\begin{array}{c}\text { S } \\
\text { SVM } \\
(\%)\end{array}$ & $\begin{array}{c}\text { Sp } \\
\text { kNN } \\
(\%)\end{array}$ & $\begin{array}{c}\text { Sp } \\
\text { SVM } \\
(\%)\end{array}$ \\
\hline 4,8 & 90.63 & 93.96 & 89.58 & 92.50 & 91.67 & 95.42 \\
\hline 12,16 & 92.71 & 94.58 & 92.08 & 93.75 & $\mathbf{9 3 . 3 3}$ & 95.42 \\
\hline $4,12,16$ & $\mathbf{9 2 . 9 2}$ & 93.96 & 92.92 & 93.75 & 92.92 & 94.17 \\
\hline $4,8,12,16$ & 92.08 & $\mathbf{9 7 . 2 9}$ & 91.25 & 96.25 & 92.92 & $\mathbf{9 8 . 3 3}$ \\
\hline All & 90.21 & 97.08 & $\mathbf{9 4 . 5 8}$ & $\mathbf{9 7 . 5 0}$ & 85.83 & 96.67 \\
\hline
\end{tabular}

Each EEG channel reports the activity of the brain region where it is located. A focal crisis originating in the occipital lobe has clinical manifestations that reflect this region of the brain (Shoeb, 2009). Occipital crises are usually triggered by visual stimulation, such as induction by television and by the closure of the eyes (Yalçin, Kaymaz and Forta, 2000).

During data collection, patients with epilepsy were used who could present focal, lateral or generalized seizures (Shoeb et al., 2004). Therefore, since the most relevant channels to discriminate the interictal and ictal states are in the occipital lobe region, it cannot be said that patients have focal epilepsy. This is likely, because focal seizures can also be spread to other regions of the brain (Shoeb, 2009).

From the results, we can detect the preictal state from the occipital lobe region, with only 4 channels, considering the patient's type of epilepsy. 
Therefore, it is possible to construct a non-invasive device that works using 4 channels that can be located discretely in the posterior region of the head.

\subsection{Analysis by sub-bands obtained by DWT}

After the channel analysis step, the classification for each sub-band obtained by DWT, using only channels 4, 8, 12 and 16 , was performed to verify the frequency bands that are most important to distinguish between the interictal and preictal states. The Table 4 shows the results of each subband for each classifier.

Table 4. Performance of the $\mathrm{kNN}$ and SVM classifiers in relation to the sub-bands

\begin{tabular}{|c|c|c|c|c|c|c|}
\hline Sub-bands & $\begin{array}{c}\text { Acc } \\
\text { kNN } \\
(\%)\end{array}$ & $\begin{array}{c}\text { Acc } \\
\text { SVM } \\
(\%)\end{array}$ & $\begin{array}{c}\text { S } \\
\text { kNN } \\
(\%)\end{array}$ & $\begin{array}{c}\text { S } \\
\text { SVM } \\
(\%)\end{array}$ & $\begin{array}{c}\text { Sp } \\
\text { kNN } \\
(\%)\end{array}$ & $\begin{array}{c}\text { Sp } \\
\text { SVM } \\
(\%)\end{array}$ \\
\hline D1 & 86.25 & 78.13 & 83.75 & 78.33 & $\mathbf{8 8 . 7 5}$ & 77.92 \\
\hline D2 & 85.42 & 83.75 & 90.00 & 79.17 & 80.83 & 88.33 \\
\hline D3 & $\mathbf{8 9 . 5 8}$ & $\mathbf{8 9 . 7 9}$ & $\mathbf{9 1 . 6 7}$ & $\mathbf{8 7 . 5 0}$ & 87.50 & 92.08 \\
\hline D4 & 76.25 & 77.50 & 80.83 & 86.25 & 71.67 & 68.75 \\
\hline D5 & 83.33 & 80.42 & 82.92 & 75.00 & 83.75 & 85.83 \\
\hline A5 & 83.75 & 82.29 & 86.67 & 69.17 & 80.83 & $\mathbf{9 5 . 4 2}$ \\
\hline
\end{tabular}

From Table 4 we observed that D1 (64-128 Hz), D2 (32-64 $\mathrm{Hz})$ and $\mathrm{D} 3(16-32 \mathrm{~Hz})$ presented better relevance in the classification. When the D4 $(8-16 \mathrm{~Hz}), \mathrm{D} 5(4-8 \mathrm{~Hz})$ and the approximation $(0-4 \mathrm{~Hz})$ levels were removed from the analyze, the classifiers achieved results around $95 \%$ for all metrics.

Table 5. Performance of the KNN and SVM classifiers in relation to the sub-bands D1, D2 and D3

\begin{tabular}{|c|c|c|c|c|c|c|}
\hline Sub-bands & $\begin{array}{c}\text { Acc } \\
\text { kNN } \\
(\%)\end{array}$ & $\begin{array}{c}\text { Acc } \\
\text { SVM } \\
(\%)\end{array}$ & $\begin{array}{c}\text { S } \\
\text { kNN } \\
(\%)\end{array}$ & $\begin{array}{c}\text { S } \\
\text { SVM } \\
(\%)\end{array}$ & $\begin{array}{c}\text { Sp } \\
\text { kNN } \\
(\%)\end{array}$ & $\begin{array}{c}\text { Sp } \\
\text { SVM } \\
(\%)\end{array}$ \\
\hline D1, D2, D3 & 94.58 & 95.83 & 95.42 & 95.42 & 93.75 & 96.25 \\
\hline
\end{tabular}

Therefore, the range of $32 \mathrm{~Hz}$ to $128 \mathrm{~Hz}$ presented greater relevance for a classification of the interictal and preictal states. While studies related to the detection of the crisis, that is, in the discrimination of the interictal and ictal state, have considered the bands with lower frequencies as the entry of the classifier (Li, Chen and Zhang, 2017; Kaleem, Guergachi and Krishnan, 2018). Since most of the convulsive activity occurs in the range of $3 \mathrm{~Hz}$ to $29 \mathrm{~Hz}$ (Kaleem, Guergachi and Krishnan, 2018). This implies that higher frequencies contribute more to a good classification of the interictal and preictal states and can be analyzed for the precursors of the seizures (Gadhoumi, Lina and Gotman, 2012).

\section{CONCLUSION}

The understanding of how epileptic seizures are generated and if they are predictable is of paramount importance in developing new research that can identify preictal and interictal activities in EEG signals. Thus, discrimination of states between convulsions and before seizures contributes to the construction of an efficient prediction and alert system of epileptic seizures (Song, Zhang, 2016).

Machine learning has made a lot of contribution to the methodologies applied in predicting epileptic seizures due to the complexity of EEG signals. Thus, it has been facilitated the evaluations of the multivariate analyzes and of the feature spaces to differentiate the characteristics between preictal and interictal periods (Tsiouris et al., 2018).

In this work we present a methodology of discrimination of the interictal and preictal states from the study of the frequency bands obtained by DWT, and the channels that are most relevant to reach a good classification.

The database used in this work, CHB-MIT database, has been used in other researches, but few authors have used it for the same purpose presented in this paper. In addition, the high variety of patients and the presence of physiological artifacts present in the data may interfere with the results obtained (Kaleem, Guergachi and Krishnan, 2018). However, our results were favorable for a future application in seizure predictions. The availability of an algorithm, coupled with a device capable of preventing seizures, will avoid the difficulties and concerns associated with the consequences of epilepsy.

Due to the need for an efficient method for all patient types, more research is still needed. These considerations motivate more analyses in order to find out methods that can be generalized among patients of different genders and ages.

As an extension of our work, we intend to use a larger data set with greater diversity among patients. In addition, we intend to apply methods to predict the epileptic seizure.

\section{ACKNOWLEDGMENTS}

The authors thank the Programa de Pós-Graduação em Engenharia Elétrica (PPGEE - UFES) and the financial support for the research from the project of the Fundação de Amparo à Pesquisa do Espírito Santo (FAPES), number 598/2018. Jade B. Kill thanks for FAPES/CAPES (Edital 02/2018) for her grant.

\section{REFERENCES}

Burges, C. J.c. (1998). A Tutorial on Support Vector Machines for Pattern Recognition. Data Mining And Knowledge Discovery, v. 2, p.121-167.

Chu, H. et al. (2017). Predicting epileptic seizures from scalp EEG based on attractor state analysis. Computer Methods And Programs In Biomedicine, v. 143, p.75-87.

Cover, T., Hart, P. (1967). Nearest neighbor pattern classification. IEEE Transactions on Information Theory, v. 13, p. 21-27. 
Faust, O. et al. (2015). Wavelet-based EEG processing for computer-aided seizure detection and epilepsy diagnosis. Seizure, v. 26, p.56-64.

Freestone, D.R., Karoly, P.J., Cook, M.J. (2017). A forwardlooking review of seizure prediction. Current Opinion In Neurology, v. 30, p.167-173.

Gadhoumi, K., Lina, J., Gotman, J. (2012). Discriminating preictal and interictal states in patients with temporal lobe epilepsy using wavelet analysis of intracerebral EEG. Clinical Neurophysiology, v. 123, p.1906-1916.

Gubner, J. A., Chang, W. (1995). Wavelet transforms for discrete-time periodic signals. Signal Processing, v. 42, p.167-180.

Kaleem, M., Guergachi, A., Krishnan, S. (2018). Patientspecific seizure detection in long-term EEG using wavelet decomposition. Biomedical Signal Processing And Control, v. 46, p.157-165.

Khan, Y., Gotman, J. (2003). Wavelet based automatic seizure detection in intracerebral electroencephalogram. Clinical Neurophysiology, v. 114, p.898-908.

Kiral-Kornek, I. et al. (2018) Epileptic Seizure Prediction Using Big Data and Deep Learning: Toward a Mobile System. Ebiomedicine, v. 27, p.103-111.

Kocadagli, O., Langari, R. (2017). Classification of EEG signals for epileptic seizures using hybrid artificial neural networks based wavelet transforms and fuzzy relations. Expert Systems With Applications, v. 88, p.419-434.

Kohavi, R. (1995). A Study of Cross-Validation and Bootstrap for Accuracy Estimation and Model Selection. Proceedings of the 14th International Joint Conference on Artificial Intelligence, v. 2, p. 1137-1145.

Li, M., Chen, W., Zhang, T. (2017). Classification of epilepsy EEG signals using DWT-based envelope analysis and neural network ensemble. Biomedical Signal Processing And Control, v. 31, p.357-365.

Mormann, F. et al. (2007) Seizure prediction: the long and winding road. Brain, v. 130, p.314-333.

Richhariya, B., Tanveer, M. (2018). EEG signal classification using universum support vector machine. Expert Systems With Applications, v. 106, p.169-182.

Saab, M.e.; Gotman, J. (2005). A system to detect the onset of epileptic seizures in scalp EEG. Clinical Neurophysiology, v. 116, p.427-442.

Sayeid, M.I.E. et. al. (2016). Statistical analysis of EEG signals in wavelet domain for efficient seizure prediction, American Journal of Biomedical Engineering, v. 6, p. 32-41.

Sharif, B., Jafari, A.H. Prediction of epileptic seizures from EEG using analysis of ictal rules on Poincaré plane. (2017). Computer Methods and Programs in Biomedicine, v. 145, p.11-22.

Shoeb, A. et al. (2004). Patient-specific seizure onset detection. Epilepsy \& Behavior, v. 5, p.483-498.

Shoeb, A. (2009) Application of Machine Learning to Epileptic Seizure Onset Detection and Treatment. PhD Thesis of Massachusetts Institute of Technology, p.1-162.

Song, Y., Zhang, J. (2016). Discriminating preictal and interictal brain states in intracranial EEG by sample entropy and extreme learning machine. Journal of Neuroscience Methods, v. 257, p.45-54.

Truong, N.D. et al. (2018). Convolutional neural networks for seizure prediction using intracranial and scalp electroencephalogram. Neural Networks, v. 105, p.104111.

Tsiouris, K. M. et al. (2018). A Long Short-Term Memory deep learning network for the prediction of epileptic seizures using EEG signals. Computers In Biology And Medicine, v. 99, p.24-37.

Usman, S.M., Usman, M., Fong, S. (2017). Epileptic Seizures Prediction Using Machine Learning Methods. Computational and Mathematical Methods in Medicine, v. 2017, p.1-10.

Williamson, J.R. et al. (2012). Seizure prediction using EEG spatiotemporal correlation structure. Epilepsy \& Behavior, v. 25, p.230-238.

WHO - World Health Organization. (2019). Epilepsy. Available at: https://www.who.int/news-room/factsheets/detail/epilepsy (accessed 4 April 2019).

Yalçin, A. D., Kaymaz, A., Forta, H. (2000) Reflex occipital lobe epilepsy. Seizure, v. 9, p.436-441. 